\title{
Growth and formation of InGaN and GaN nano-structures studied by STM*
}

\author{
Subhashis Gangopadhyay ${ }^{\dagger}$, Thomas Schmidt, Sven Einfeldt ${ }^{\ddagger}$, Tomohiro Yamaguchi, Detlef Hommel and Jens Falta \\ Institute of Solid State Physics, University of Bremen, \\ Otto-Hahn-Allee 1, 28359 Bremen, Germany
}

(Received 18 October 2005; Accepted 14 December 2005; Published 19 January 2006)

\begin{abstract}
Growth and morphology of metal organic vapour phase epitaxy (MOVPE) deposited InGaN nano-islands and molecular beam epitaxy (MBE) grown GaN films on GaN(0001) template layers on sapphire substrates have been investigated using scanning tunneling microscopy. For MOVPE InGaN growth, the nucleation of self-organized nano-structures can be achieved by a careful choice of the growth temperature, the In partial pressure, the growth rate and V/III flux ratio. For growth at $650^{\circ} \mathrm{C}$, large spiral disc-like islands are found, preferentially nucleating at GaN substrate defects. At $600^{\circ} \mathrm{C}$, islands of smaller average size are observed. Lowering the In flux at this temperature, a homogeneous nucleation of small quantum dot like islands with a density of $10^{12} / \mathrm{cm}^{2}$ is found. For homoepitaxial MBE growth of thin GaN layers on GaN templates, a layer-by-layer growth mode is observed for Ga rich growth conditions. For growth at $750^{\circ} \mathrm{C}$, an atomically resolved $4 \times 4$ surface reconstruction with a high defect density is found in the initial growth stage. However, subsequent growth at $790^{\circ} \mathrm{C}$ leads to the formation of one dimensional nanoclusters of about $3 \mathrm{~nm}$ lateral spacing. For GaN growth at a lower Ga-flux, a rougher surface morphology and three dimensional growth is observed. Independent on the Ga flux, one-dimensional nanostructures appear after prolonged growth at higher temperature, which are attribute to the impact of ions emerging from the N-plasma. [DOI: 10.1380/ejssnt.2006.90]
\end{abstract}

Keywords: Scanning Tunneling Microscopy; Gallium nitride; InGaN QDs;

\section{INTRODUCTION}

GaN nitride based wide-bandgap materials have received high attention due to their applications in laser diodes (LD) or light emitting diodes (LED) [1, 2]. Particularly, $\operatorname{In}_{x} \mathrm{Ga}_{1-x} \mathrm{~N}$ alloys have attracted broad interest due to their use as active layers. Much work has been devoted to $\operatorname{In}_{x} \mathrm{Ga}_{1-x} \mathrm{~N} /$ GaN quantum wells (QWs), which allowed for the commercialization of light emitting diodes (LEDs) and laser diodes (LDs) in the blue spectral region [1]. Nanometer-scale fluctuations of the In mole fraction have been found within the InGaN QW structures, [3$6]$ and theoretical calculations predict a high tendency of phase separation within InGaN alloys [7]. The indiumrich phases in the $\operatorname{In}_{x} \mathrm{Ga}_{1-x} \mathrm{~N}$ film may act as centers for carrier localization and exhibit a quantum dot (QD)-like behavior $[5,6]$. For better device performance the implementation of quantum dot (QD) structures is promising as it prevents the carriers from nonradiative recombination at crystal defects $[8,9]$.

A prerequisite for the successful growth of InGaN QDs on GaN substrates is a better understanding of the underlying growth mechanisms. As the growth properties of subsequently grown InGaN structures are closely related to the GaN substrate properties, a thorough characterization of the GaN surface is also of high interest. Apart from the growth of InGaN QDs, today's nitride based devices are mostly grown on GaN template layers, which are epitaxially prepared on hetero-substrates as e.g. SiC and

${ }^{\dagger}$ Corresponding author: subha@ifp.uni-bremen.de

¥Present address: Ferdinand-Braun-Institut für Höchstfrequenztechnik, Gustav-Kirchhoff-Strasse 4, 12489 Berlin, Germany

* This paper was presented at International Symposium on Surface Science and Nanotechnology (ISSS-4), Saitama, Japan, 14-17 November, 2005. sapphire.

Within this work, growth and morphology of metal organic vapour phase epitaxy (MOVPE) grown InGaN nano-islands on GaN templates have been investigated using scanning tunneling microscopy. Additionally, the surface morphology of molecular beam epitaxy (MBE) grown thin GaN films on MOVPE template layers have also been studied.

\section{EXPERIMENTAL}

The InGaN deposition was performed on $\mathrm{GaN}(0001)$ template layers grown on c-plane sapphire substrates using atmospheric pressure MOVPE which was carried out in a vertical type showerhead reactor (Thomas Swan) using $\mathrm{N}_{2}$ as a carrier gas. Trimethyl-gallium (TMGa), trimethyl-indium (TMIn) and ammonia $\left(\mathrm{NH}_{3}\right)$ were employed as $\mathrm{Ga}$, In and $\mathrm{N}$ sources, respectively. GaN growth for template layers was optimized at $1050^{\circ} \mathrm{C}$. For subsequent InGaN growth, basically three different growth conditions were used: (1) and (2) growth at $650^{\circ} \mathrm{C}$ and $600^{\circ} \mathrm{C}$, respectively, with high In partial pressure $[\mathrm{In}:(\mathrm{In}+\mathrm{Ga})=0.85]$ and $(3)$ growth at $600^{\circ} \mathrm{C}$ with reduced In partial pressure $[\mathrm{In}:(\mathrm{In}+\mathrm{Ga})=0.74]$. After InGaN growth, the samples were transferred under dry nitrogen ambient to the scanning tunneling microscopy (STM) analysis chamber. Prior to the STM experiments, the samples were thermally degassed under ultra-high vacuum (UHV) at $500^{\circ} \mathrm{C}$.

In case of thin GaN layer deposition, the $\mathrm{GaN}(0001) /$ sapphire templates were also transferred under $\mathrm{N}_{2}$ ambient to the combined STM and MBE growth chamber and thermally degassed at $700^{\circ} \mathrm{C}$ before the growth experiments. A radio frequency $\mathrm{N}$-plasma was used as an active nitrogen source and an electron beam evaporator was employed as a Ga source. Two growth regimes were selected: (1) growth with high 

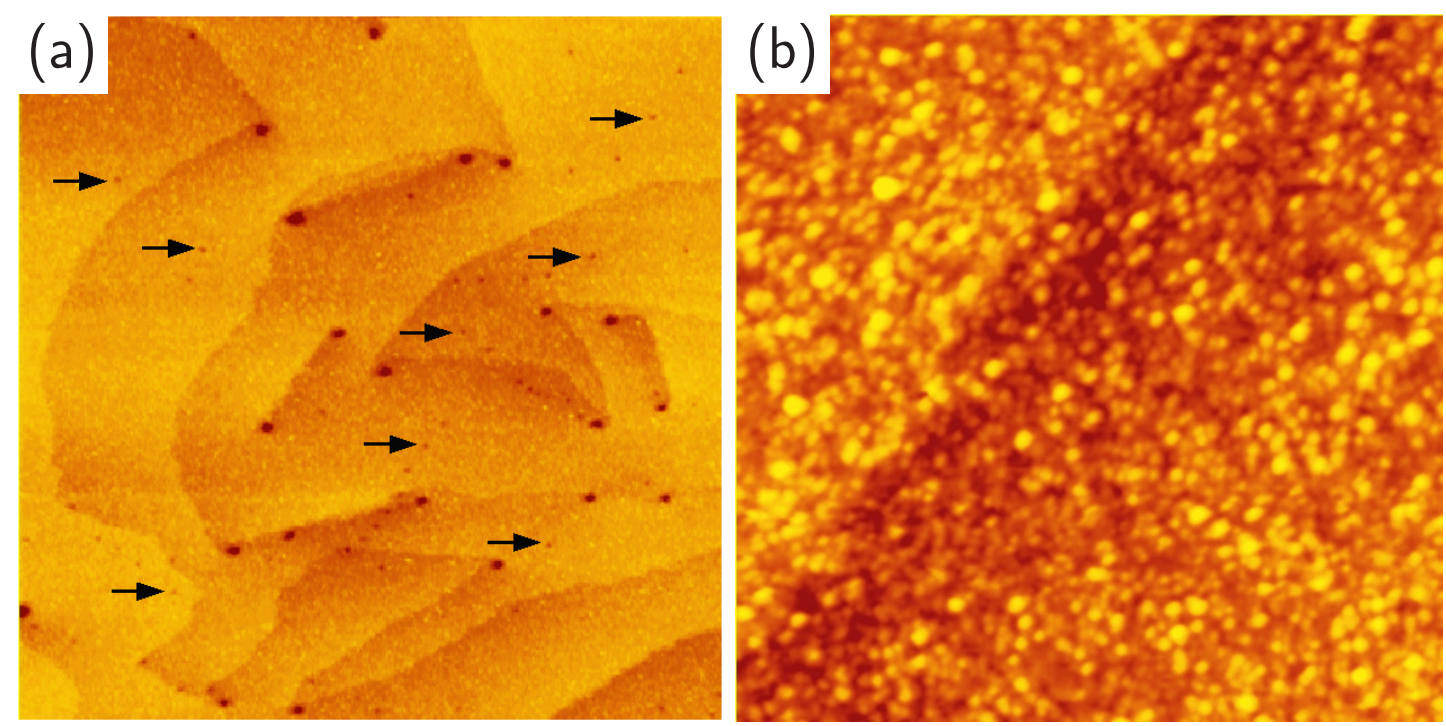

FIG. 1: Empty state STM images of the MOVPE grown GaN(0001) layer on c-plane sapphire substrate transferred under $\mathrm{N}_{2}$ ambient. Scan areas are (a) $1000 \times 1000 \mathrm{~nm}^{2}$ and (b) $100 \times 100 \mathrm{~nm}^{2}$, respectively.
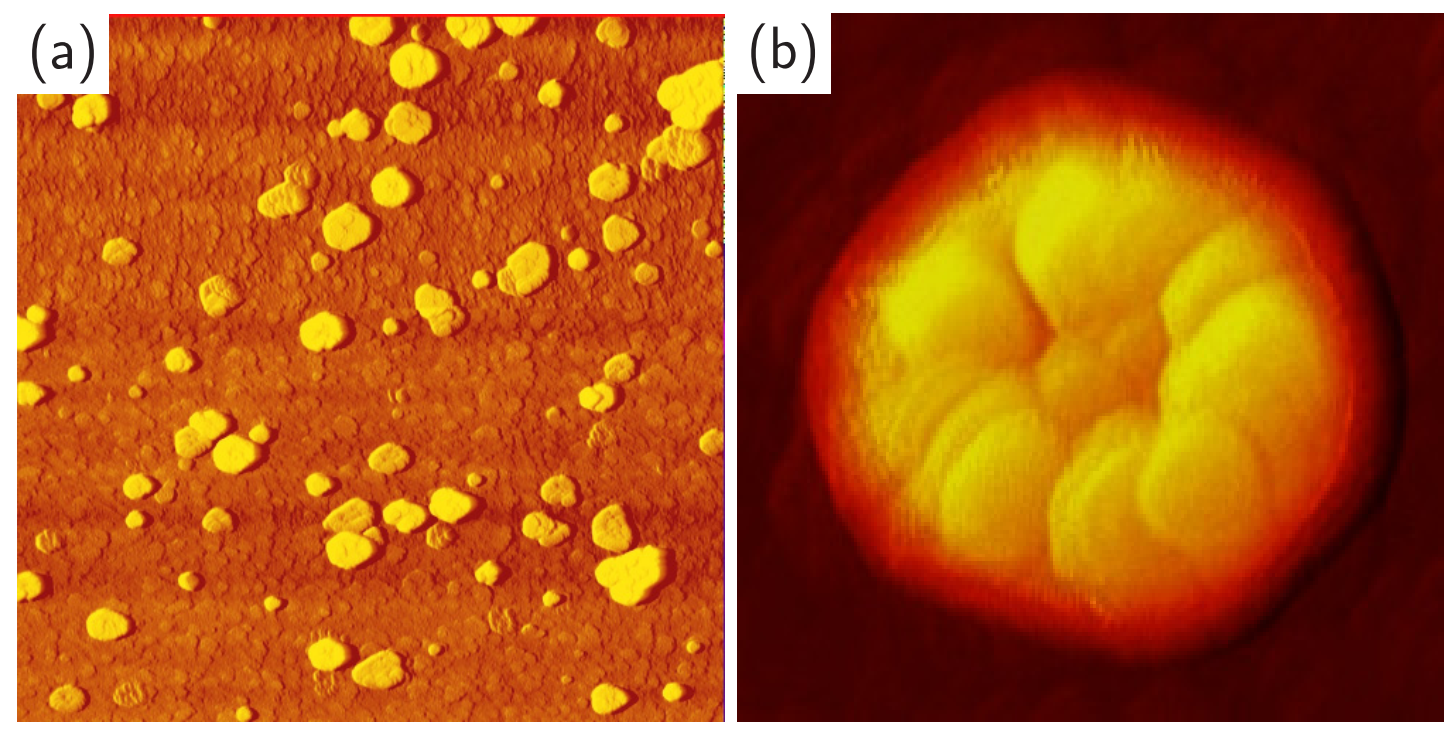

FIG. 2: Empty state STM images of MOVPE grown $1.3 \mathrm{~nm} \mathrm{InGaN} \mathrm{layer} \mathrm{on} \mathrm{GaN} \mathrm{template} \mathrm{for} \mathrm{In-rich} \mathrm{growth} \mathrm{at} 650^{\circ} \mathrm{C}$. Scan areas are for (a) $1000 \times 1000 \mathrm{~nm}^{2}$ and (b) $100 \times 100 \mathrm{~nm}^{2}$, respectively.

Ga flux (slightly Ga-rich growth) and (2) growth with reduced Ga flux (N-rich growth). The MBE chamber has an in-situ sample transfer facility to the STM analysis chamber. All STM characterization and MBE growth was performed under UHV with a base pressure below $10^{-10}$ mbar. The temperatures were measured by an infrared pyrometer with an absolute uncertainty of about $\pm 25^{\circ}$. All STM measurements presented in this paper were performed under constant current mode imaging conditions.

\section{RESULTS AND DISCUSSION}

\section{A. $\mathrm{GaN}(0001) /$ sapphire template}

The typical surface morphology of MOVPE grown GaN template layers is shown in figure $1(\mathrm{a})$. The GaN film is quite smooth consisting of an array of terraces separated by steps of $\sim 2-3 \AA$ height, well in agreement with the monolayer step height (2.6 $\AA$ for GaN(0001)). Most of the steps are terminated tangentially at the edges of surface pits/depressions (large black dots). This type of pits can be correlated to screw or mixed-type surface dislocations, which are quite common for GaN template layers grown on sapphire. Apart from the screw or mixed-type surface dislocations, some smaller and shallower pits/depressions (small faint-dark spots marked by arrows) can also be found which are located within the GaN terrace areas. These smaller pits are correlated to pure edge type dislocations of GaN. Since the Burgers vector of pure edge dislocations does not have a component perpendicular to the surface, no steps are generated or terminated. In a high resolution STM image of the GaN surface small granular islands are observed as shown in figure 1(b) and the surface structure cannot be resolved atomically. 


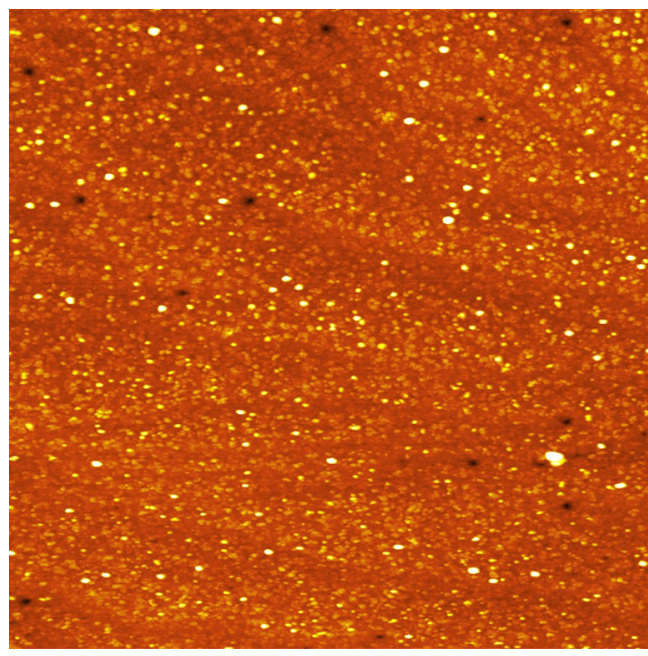

(a)

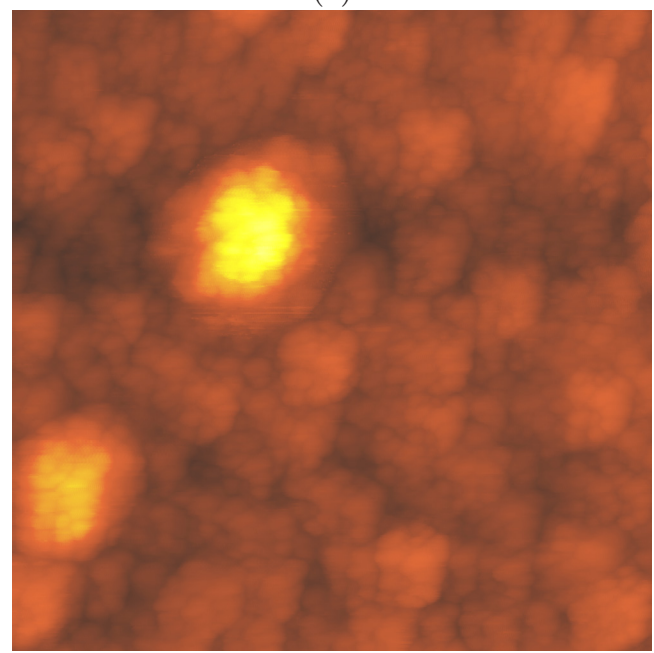

(b)

FIG. 3: Empty state STM images of MOVPE grown $1.3 \mathrm{~nm}$ InGaN layer on GaN template for In-rich growth condition at $600^{\circ} \mathrm{C}$. Scan areas are for (a) $1000 \times 1000 \mathrm{~nm}^{2}$ and (b) $100 \times 100$ $\mathrm{nm}^{2}$, respectively.

\section{B. InGaN growth on $\mathrm{GaN}(0001)$ templates using MOVPE}

As already mentioned, three different parameter sets for the MOVPE growth conditions were used for InGaN growth on $\mathrm{GaN}(0001)$ templates. In case of InGaN growth with high In partial pressure $[\mathrm{In}:(\mathrm{In}+\mathrm{Ga})=0.85]$, the $\mathrm{V}-$ III ratio was fixed at $2.2 \times 10^{3}$ and a growth rate of 0.26 $\mathrm{nm} / \mathrm{sec}$ was measured by cross-sectional scanning electron microscopy measurements of thick InGaN reference layers. For InGaN growth with reduced In partial pressure $[\mathrm{In}:(\mathrm{In}+\mathrm{Ga})=0.74]$, the $\mathrm{V}-\mathrm{III}$ ratio was increased to $3.9 \times 10^{3}$ and the effective growth rate was reduced by a factor of two $(0.13 \mathrm{~nm} / \mathrm{sec})$.

\section{In GaN growth at $650^{\circ} \mathrm{C}$ with high In partial pressure}

The surface morphology after deposition of $1.3 \mathrm{~nm}$ In$\mathrm{GaN}$ at $650^{\circ} \mathrm{C}$ with high In partial pressure is shown in figure 2 (a). Two types of large InGaN islands are found:

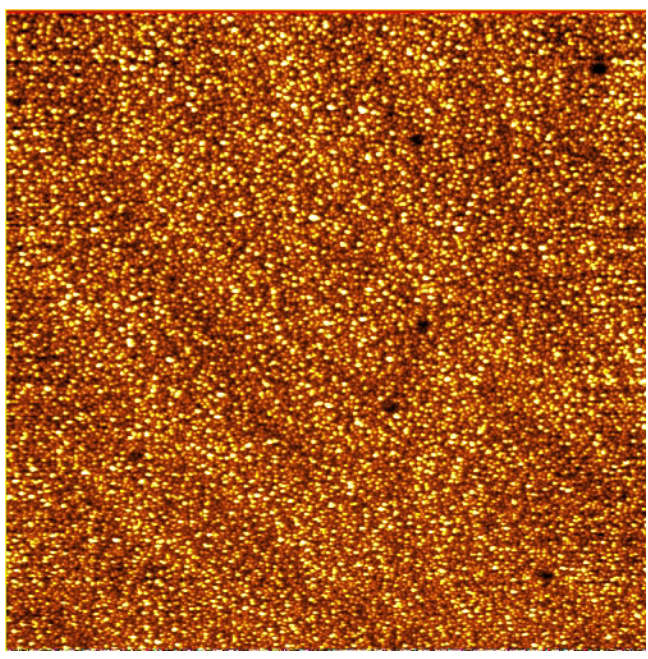

(a)

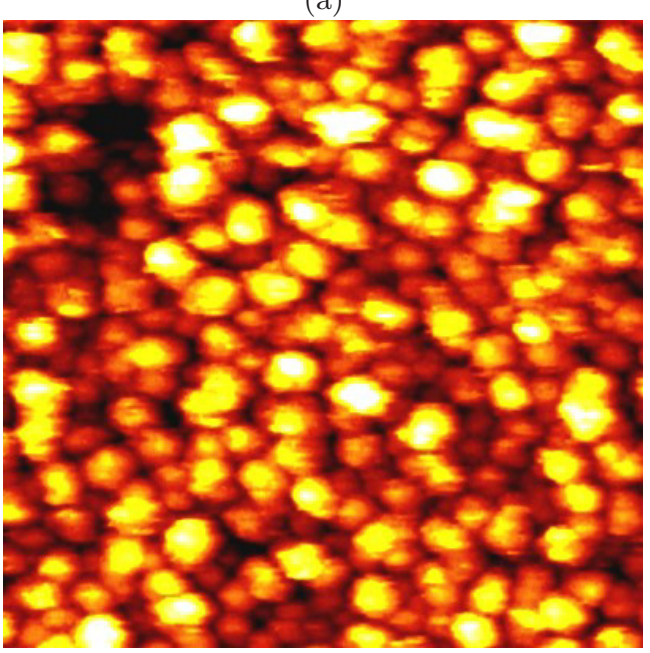

(b)

FIG. 4: Empty state STM images of the MOVPE grown 3.9 $\mathrm{nm}$ InGaN layer for low In-flux at $600^{\circ} \mathrm{C}$. Scan areas for (a) $1000 \times 1000 \mathrm{~nm}^{2}$ and (b) $100 \times 100 \mathrm{~nm}^{2}$, respectively.

(i) islands with a spiral arrangement of discs (diameter $d \approx 80 \mathrm{~nm}$, height $h \approx 10 \mathrm{~nm}$ ) and (ii) islands with a flat top facet $(d \approx 30 \mathrm{~nm}, h \approx 3 \mathrm{~nm})$. The wetting layer of the InGaN surface appears very smooth and no defects attributed to the screw / mixed type dislocations of the GaN substrate (dark spots in figure 1(a)) are visible here. The large spiral disc-like islands have a very specific structure which is different from those reported for spiral InGaN islands [11]. Here, the islands appear in a distorted hexagonal shape with a central pit. The individual "discs" are atomically flat and separated from each other by steps of monolayer height. A close-up of a spiral disclike island is shown in figure 2 (b). In case of larger InGaN deposits, In droplets are found, preferentially located on top of the large islands (images not shown here).

The finding of a smooth wetting layer without any visible defects after InGaN growth and the spiral stacking of island discs with a central pit indicate the preferential nucleation of large islands at the defect sites of the GaN template, more precisely, at or around the screw/mixedtype dislocations. The density of the spiral disc-like islands is also close to the defect density observed for the 

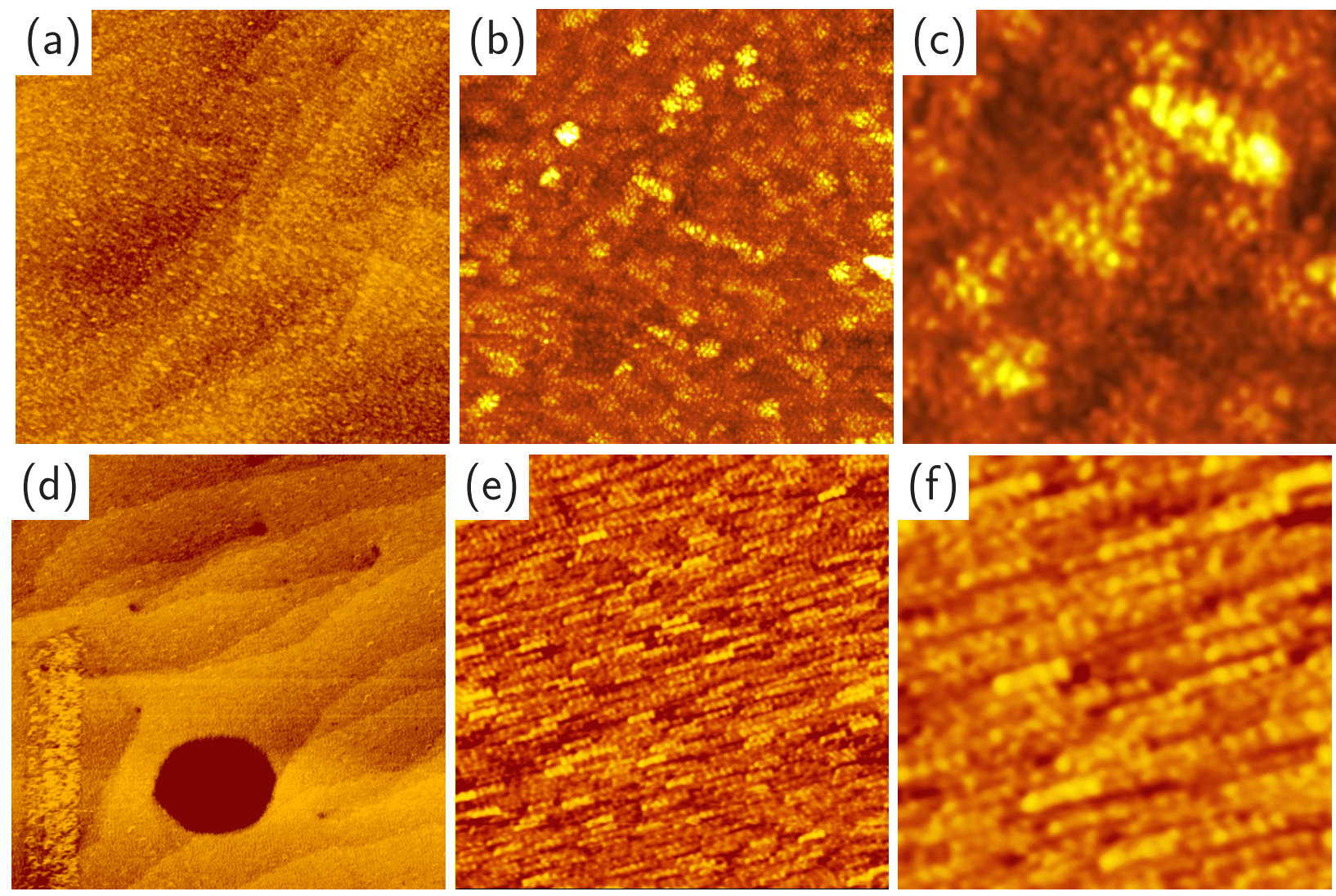

FIG. 5: STM images after MBE deposition of about $4 \mathrm{~nm}$ of $\mathrm{GaN}$ deposition at $750^{\circ} \mathrm{C}$ on $\mathrm{GaN} /$ sapphire template: (a) $1000 \times 1000$ $\mathrm{nm}^{2}$, (b) $150 \times 150 \mathrm{~nm}^{2}$ and (c) $40 \times 40 \mathrm{~nm}^{2}$. After further deposition of about another $4 \mathrm{~nm}$ of GaN at $790^{\circ} \mathrm{C}:(\mathrm{d}) 1000 \times 1000$ $\mathrm{nm}^{2}$, (e) $200 \times 200 \mathrm{~nm}^{2}$ and (f) $70 \times 70 \mathrm{~nm}^{2}$, respectively.

GaN templates, as expected for the case of defect induced InGaN island nucleation. This effect can be explained in terms of the thermal diffusion length of the deposited material at this temperature being longer than the average defect spacing. The hexagonal shape of the spiral islands can be attributed to the six-fold crystal symmetry and the atomically flat (0001) top facet on the large islands reflect the minimization of the total surface free energy.

\section{In GaN growth at $600^{\circ} \mathrm{C}$ with high In partial pressure}

By lowering the InGaN growth temperature to $600^{\circ} \mathrm{C}$ while keeping the other growth parameters fixed, a different surface morphology is obtained, as shown in figure 3 (a). Again, large islands can be found, but the average size is reduced by a factor of about $2(d \approx 35 \mathrm{~nm}$, $h \approx 3.5 \mathrm{~nm}$ ). Moreover, the wetting layer appears rougher and exhibits smaller islands $(d \approx 10 \mathrm{~nm}, h \approx 2 \mathrm{~nm})$. In contrast to growth at $650^{\circ} \mathrm{C}$, defects attributed to the GaN template are visible after InGaN deposition (dark spots in the STM image). The large islands show a rough surface morphology with a granular structure as shown in figure 3 (b). Indium droplets are not found for these growth conditions even for larger InGaN deposits.

The smaller size distribution of the large InGaN islands can be attributed to a reduced thermal diffusion of adatom species. The reduced diffusion length also impedes heterogeneous nucleation at defects. Homogeneous nucleation also explains the formation of smaller islands on the wetting layer. Moreover, a lower growth temperature will also enhance the In incorporation, in agreement with the disappearance of In droplets for thicker films. The formation of an InGaN wetting layer of larger In content will cause an increased strain leading to the formation of small islands, since the system can reduce its strain energy by a partial elastic relaxation of the InGaN in the islands.

\section{In GaN growth at $600^{\circ} \mathrm{C}$ with reduced In partial pressure}

By reducing the In-flux at $600^{\circ} \mathrm{C}$, the surface morphology changes drastically and the most uniform size distribution of InGaN islands with an average diameter of about $8 \mathrm{~nm}$ and a height of $2-3 \mathrm{~nm}$ was achieved as shown in figure 4 . The topograph in figure 4 (a) provides an overview of the InGaN surface where defects of the GaN template as well as step edges are visible but the nucleation of larger islands is not observed. In figure 4 (b), an image at larger magnification shows the homogeneously distributed QD-like InGaN nano-islands with a density of about $10^{12} \mathrm{~cm}^{-2}$. Judging from their size these nanoislands could be suitable for three-dimensional quantum confinement.

The findings of homogeneously distributed InGaN QDs can be explained in terms of reduced surface mobility which suppresses island ripening and hence opposes the formation of large islands. In addition, a reduced growth rate can also reduce the In incorporation [3] which will 


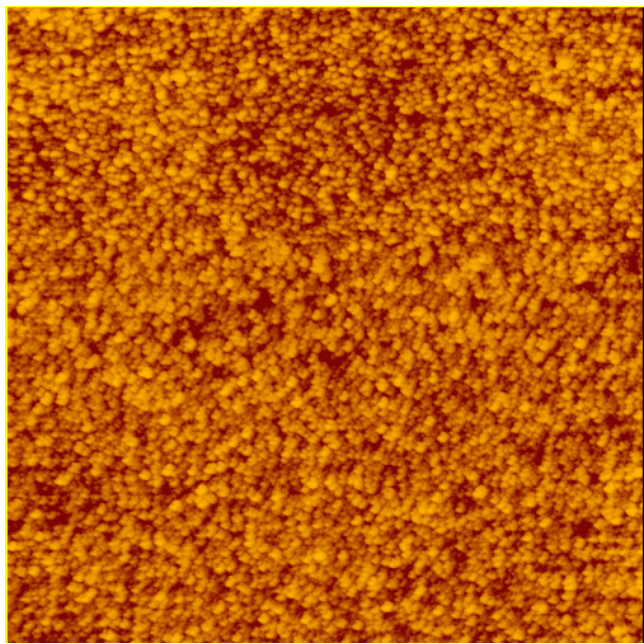

(a)

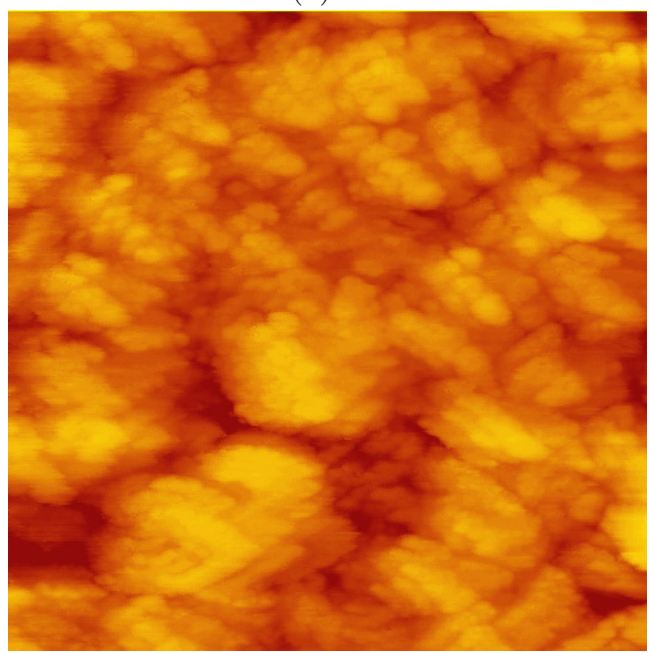

(c)

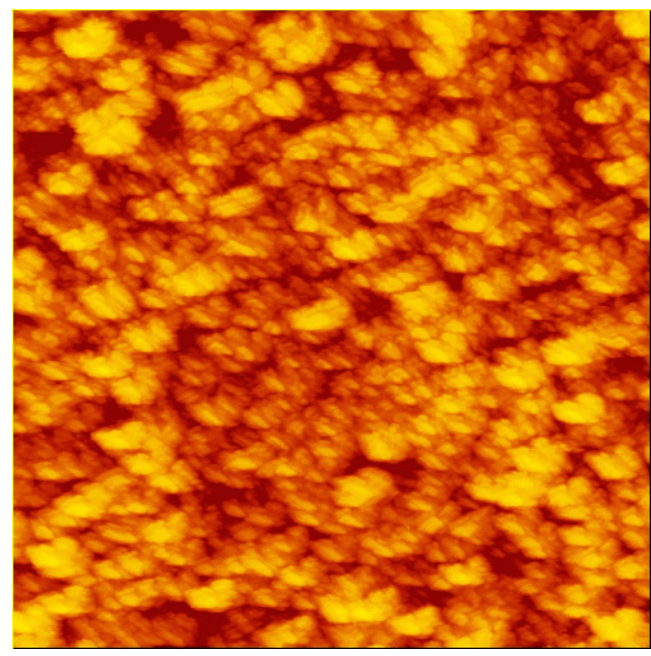

(b)

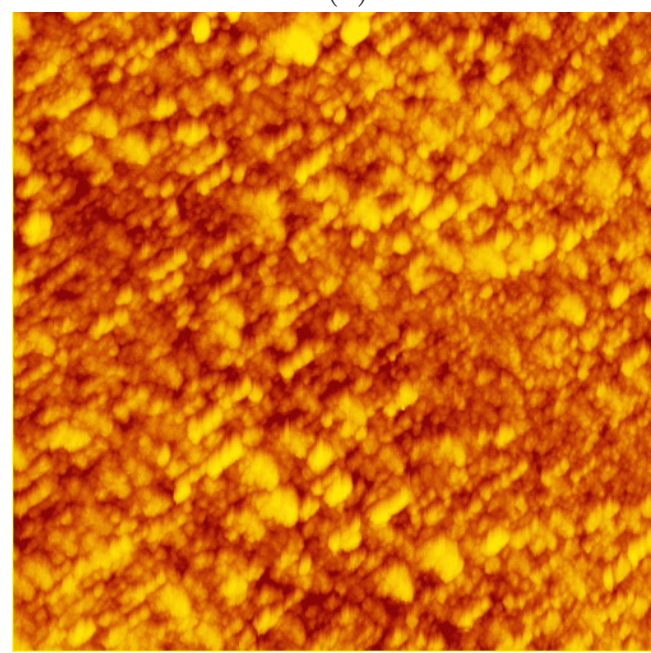

(d)

FIG. 6: STM images after MBE growth of about $4 \mathrm{~nm} \mathrm{GaN} \mathrm{at} 750^{\circ} \mathrm{C}$ with reduced Ga-flux: (a) $1000 \times 1000 \mathrm{~nm}^{2}$ $\mathrm{nm}^{2}$ and (c) $75 \times 75 \mathrm{~nm}^{2}$. After further deposition of about another $4 \mathrm{~nm} \mathrm{GaN}$ at $800^{\circ} \mathrm{C}$ : (d) $500 \times 500 \mathrm{~nm}^{2}$.

(b) $290 \times 290$

suppress 3D islanding. Moreover, the reduced In partial pressure leads to further nitrogen-enriched growth conditions and theoretical studies also predicted a smaller island size for N-rich growth conditions [10, 12].

\section{GaN growth on $\mathrm{GaN}(0001)$ templates using MBE}

In case of homoepitaxial growth of thin GaN layers on GaN(0001) templates using MBE, basically two different growth regimes were investigated with high and low $\mathrm{Ga}$ flux, respectively. For GaN growth with low Ga flux, the flux monitor current was set to $60 \%$ of the value used for the high Ga flux experiments keeping the active nitrogen flux unchanged. For each growth regime (a) the initial nucleation of about $4 \mathrm{~nm} \mathrm{GaN}$ at $750^{\circ} \mathrm{C}$ and (b) the subsequent growth of about another $4 \mathrm{~nm} \mathrm{GaN} \mathrm{at} 790-800^{\circ} \mathrm{C}$ was studied. After every growth step, the samples were annealed at the growth temperature for a few minutes to remove excess $\mathrm{Ga}$ which might form droplets.

\section{GaN growth with high Ga flux}

In figure 5, STM images of a homo-epitaxial GaN film grown at $750^{\circ} \mathrm{C}$ (figure $5(\mathrm{a})-(\mathrm{c})$ ) and $790^{\circ} \mathrm{C}$ (figure $5(\mathrm{~d})-$ (f)) with high Ga-flux are presented. The surface of the GaN films appears very smooth and the terrace/step structure typical of $\mathrm{GaN}$ templates with dislocation pits are preserved (figure $5(\mathrm{a})$ ). On the STM image in figure 5 (b) a small surface roughness and the formation of small 2D islands with a height of 2.5-5.0 $\AA$ can be observed. In figure 5 (c), an atomically resolved $4 \times 4$ surface reconstruction with high defect density is found. After subsequent GaN growth at $790^{\circ} \mathrm{C}$, the surface morphology does not change much and still appears with flat terraces and dislocation pits as shown in figure 5 (d). But at closer inspection a significant change in the surface structure is revealed as shown in figure 5 (e). A one dimensional ordering of nano-clusters of about $3 \mathrm{~nm}$ average lateral spacing is observed, which is shown at higher resolution in figure $5(\mathrm{f})$.

The smooth surface morphology with step edges and dislocation pits can be attributed to a layer-by-layer growth mode. For Ga-rich growth condition, the sur- 
plus Ga atoms enhance the surface mobility which promotes the formation of a smooth surface morphology [10]. The $4 \times 4$ surface reconstruction suggests a Ga-polar $\mathrm{GaN}(0001)$ surface which is in agreement with the earlier reported surface reconstructions. According to the structural model proposed by Xue et al. [13], the $4 \times 4$ surface reconstruction is formed by removing Ga-adatoms from $\mathrm{T}_{4}$ site of the $2 \times 2$ reconstruction. The $1 \mathrm{D}$ array of nanoclusters may be attributed to the impact of high-energy ions from the N-plasma source [14] leading to selective growth in a preferential direction, as the substrate was not rotated during GaN growth.

\section{GaN growth with reduced Ga flux}

In case of GaN growth with reduced Ga flux, the surface morphology changes and a three dimensional growth front is obtained that results in the formation of a rough surface with 3D islands of about $1 \mathrm{~nm}$ height. The step and terrace structures and the dislocation pits of GaN template disappeared for this growth condition as shown in figure 6 (a). Figure 6 (b) shows the evolution of the surface morphology after deposition of about $4 \mathrm{~nm}$ of GaN at $750^{\circ} \mathrm{C}$. The $3 \mathrm{D}$ islands consist of small terraces separated by monolayer surface steps as can be seen in the high resolution STM image (figure 6(c)). The changes of the surface morphologies after further deposition of about another $4 \mathrm{~nm} \mathrm{GaN} \mathrm{at} 800^{\circ} \mathrm{C}$ are presented in figure 6 (d). Again, 1D arrays of GaN islands are obtained which is very similar to growth with high Ga-flux at $790^{\circ} \mathrm{C}$. Also, the orientation of the GaN island alignment is identical to the nano-cluster ordering depicted in figure 5 .

The rough surface morphology and 3D growth for re- duced Ga flux is correlated to the reduced surface mobility of the adatom species. Here, the growth proceeds at rather N-rich conditions, which results in surface roughening. This finding is in good agreement with the theoretical prediction for GaN growth under N-rich growth condition [10]. The $1 \mathrm{D}$ ordering of $\mathrm{GaN}$ islands can be related to the N-plasma source induced modifications as discussed earlier. We found such an alignment of nanoclusters or islands only for high-temperature growth. This effect may be related to the GaN surface softening at high temperature which might facilitate ion induced damage/modification.

\section{CONCLUSION}

The nucleation, morphology and density of InGaN nano-islands on GaN(0001) can be controlled by the choice of proper growth conditions for MOVPE. QD-like nano-islands of high density have been achieved for InGaN growth at $600^{\circ} \mathrm{C}$ with reduced In-flux. For MBE grown GaN films, a smooth surface morphology has been observed for Ga-rich growth condition. In contrast, a tendency towards 3D island growth was encountered for growth with reduced Ga-flux.

\section{Acknowledgments}

This work was supported by the Deutsche Forschungsgemeinschaft within the framework of the Research Group Physics of nitride-based, nanostructured, light-emitting devices. (DFG grant no. FOR 506).
[1] S. Nakamura, S. Pearton and G. Fasol, The Blue Laser Diode, 2nd ed. (Springer, Berlin, 2000).

[2] I. Akasaki and H. Amano, Jpn. J. Appl. Phys., 36, 5393 (1997).

[3] F. Scholz, Prog. Crystal Growth and Charact. 35, 243 (1997).

[4] R. Singh, D. Doppalapudi, T. D. Moustakas and L. T. Romano, Appl. Phys. Lett. 70, 1089 (1996).

[5] Y. Narukawa, Y. Kawakami, M. Funato, S. Fujita, S. Fujita and S. Nakamura, Appl. Phys. Lett. 70, 981 (1997).

[6] K. P. O'Donnel, R. W. Martin and P. G. Middleton, Phys. Rev. Lett. 82, 237 (1999).

[7] I. Ho and G. B. Stringfellow, Appl. Phys. Lett. 69, 2701 (1996).

[8] K. Tachibana, T. Someya and Y. Arakawa, Appl. Phys. Lett. 74, 383 (1999).
[9] N. Grandjean and J. Massies, Appl. Phys. Lett. 72, 1078 (1998).

[10] T. Zywietz, J. Neugebauer and M. Scheffler, Appl. Phys. Lett. 73, 487 (1998).

[11] S. Keller, U. K. Mishra, S. P. DenBaars and W. Seifert, Jpn. J. Appl. Phys. 37, L431 (1998).

[12] J. Neugabauer, T. K. Zywietz, M. Scheffler, J. E. Northrup, H. Chen and R. M. Feenstra, Phys. Rev. Lett. 90, 56101 (1999).

[13] Q.-K. Xue, Q. Z. Xue, R. Z. Bakhtizin, Y. Hasegawa, I. S. T. Tsong and T. Sakurai, Phys. Rev. Lett. 82, 3074 (1999).

[14] V. Kirchner, H. Heinke, U. Birkle, S. Einfeldt, D. Hommel, H. Selke and P. L. Ryder, Phys. Rev. B 58, 15749 (1998). 